\title{
Dental Findings in the Schimke Immuno-Osseous Dysplasia
}

\author{
Marcio A. da Fonseca* \\ Department of Orthodontics and Pediatric Dentistry, University of Michigan School of Dentistry, \\ Ann Arbor, Michigan
}

Schimke immuno-osseous dysplasia is a rare autosomal recessive disorder that affects primarily bone, T lymphocytes, kidneys, and skin. The patients have a triangular face, broad nasal bridge, bulbous nose tip, small palpebral fissures, short neck, long upper lip, and low hairline. Dental abnormalities of affected patients have not been discussed in detail. The patient described in this clinical report presented with clinical and radiographic abnormalities that may constitute a diagnostic characteristic in this condition. Am. J. Med. Genet. 93:158-160, 2000. ㅇ 2000 Wiley-Liss, Inc.

\section{KEY WORDS: craniofacial abnormalities; dental manifestations; den- tinogenesis imperfecta; Schimke immuno-osseous dysplasia}

\section{INTRODUCTION}

Schimke immuno-osseous dysplasia (SIOD), or spondyloepiphyseal dysplasia, is a rare, severe, multisystem disorder that can be defined as a developmental error affecting primarily bone, T lymphocytes, kidneys, and skin [Spranger et al., 1991; Santavá et al., 1994; Lama et al., 1995; Boerkoel et al., 1998; Saraiva et al., 1999]. It appears to be inherited as an autosomal recessive trait, and its pathogenesis is not clear [Spranger et al., 1991; Santavá et al., 1994; Lama et al., 1995; Ludman et al., 1993; Bayer et al., 1998; Boerkoel et al., 1998; Saraiva et al., 1999]. The affected patients usually present intrauterine growth retardation, growth below the third percentile, and short stature with the trunk being more severely affected than the limbs [Spranger et al., 1991; Ludman et al., 1993; Hashimoto et al., 1994; Santavá et al., 1994; Lama et

*Correspondence to: Dr. Marcio A. da Fonseca, Assistant Clinical Professor, Department of Orthodontics and Pediatric Dentistry, University of Michigan School of Dentistry, 1011 N. University Avenue, Ann Arbor, MI 48109. E-mail: marcio@umich.edu

Received 9 December 1999; Accepted 13 March 2000 al., 1995; Schmidt et al., 1997; Bayer et al., 1998]. Boerkoel et al. [1998] emphasized the presence of vascular abnormalities that cause significant morbidity and mortality in patients with SIOD. There appears to be two forms of the dysplasia [Boerkoel et al., 1998]. One has a juvenile onset of growth failure and renal dysfunction in which the affected individuals survive into adulthood with renal dialysis or transplantation. The other form has infancy to early childhood onset with death occurring usually within the first decade of life. Intelligence is normal in most cases [Ludman et al., 1993; Bayer et al., 1998; Boerkoel et al., 1998; Saraiva et al., 1999], but neurological symptoms and behavioral disturbances, such as automutilation, have been reported [Schmidt et al., 1997]. Although the patients have characteristic facial changes such as a triangular face, broad nasal bridge, bulbous nose tip, small palpebral fissures, short neck, long upper lip, and low hairline, only one report has a dental anomaly [Ludman et al., 1993]. This manuscript describes a patient with Schimke immuno-osseous dysplasia who has unusual dental findings that, if determined to be common in the disorder, could facilitate its diagnosis.

\section{CLINICAL REPORT}

A 6-year-old boy presented to the Pediatric Dentistry Clinic at the University of Michigan Medical Center for a pre-bone marrow transplant oral/dental evaluation. He was born with intrauterine growth retardation 6 weeks prematurely and experienced chronic vomiting and failure to thrive by age 15 months. Café-au-lait spots were present in several areas of his body. He had undescended testes, right-crossed fused renal ectopia, proteinuria, and mild hypertension. Endocrine and gastrointestinal findings were normal. In November 1995, he was referred to the hematology clinic for evaluation of leukopenia. Results of all tests performed, including a bone marrow biopsy, were normal. Cytogenetic analysis of the bone marrow aspirate showed del(16)(q24). His neutropenia was stable until August 1996 when the absolute neutrophil count dropped to 200 , which responded well to granulocyte colony-stimulating factor therapy. In February 1997, the patient underwent bilateral orchidopexy, meatoplasty, and an open renal biopsy, which showed mild focal mesangial proliferation with hyaline thrombi and subendothelial hyaline deposits. In May 1997, skeletal 
radiographs documented bony dysplasia and mild subluxation of the hips. He had good development and range of motion, thus, no treatment was prescribed at the time. In the fall of 1997, he experienced worsening of his neutropenia, which led to an allogeneic BMT, his brother being a full-match donor. He was conditioned with busulfan and cyclophosphamide and received tracolimus and methotrexate for graft-vs-host disease prophylaxis. In October 1999, he received a renal transplant.

Examination demonstrated a broad nasal bridge and a bulbous tip of the nose. The intraoral soft tissues were unremarkable. The patient was in the mixed dentition stage and had several carious lesions. His teeth presented a gray-yellowish discoloration and appeared normal in size with minimal attrition. A hypoplastic area on the upper left first permanent molar was evident. The panoramic film (Fig. 1) documented bulbous crowns with a marked cervical constriction of the primary and permanent molars. The pulp chambers were either small in size or obliterated. The roots appeared thinner than normal. All permanent teeth were present, except for the third molars. Dental age was appropriate for chronological age. During dental treatment, it was noted that both the enamel and dentin were softer than usual. Based on his clinical and radiographic findings, a tentative diagnosis of dentinogenesis imperfecta (DI) type II was made.

\section{DISCUSSION}

This patient had all of the manifestations of Schimke immuno-osseous dysplasia, with bones, T lymphocytes, skin, and kidneys involved. Affected patients also may have fine hair and a high-pitched voice [Spranger et al., 1991; Ludman et al., 1993; Schmidt et al., 1997; Bayer et al., 1998], which were not particularly evident in this case. The skeletal surveys usually show dysplastic changes of the pelvis, proximal aspects of the femora, and the vertebral bodies [Spranger et al., 1991]. Because of the hypoplasia of the odontoid process, there is a risk of atlantoaxial dislocation and compression of the spinal cord during endotracheal intubation [Jones et al., 1980]. Mogera and Muralidhar [1996] reported that the facial characteristics of the disorder may make laryngoscopic exposure of the vocal cords difficult.

Neutropenia is usually observed by the end of the third year of life, putting the patients at risk for infectious complications [Spranger et al., 1991; Ludman et al., 1993]. No history of oral infections was reported by the patient's parents, and the oral examination showed normal soft tissues. Before the BMT, he had been admitted to the hospital only once for neutropenic fever. Renal disease in these patients is probably a secondary phenomenon [Spranger et al., 1991]. It is speculated that the defective T-cell function could predispose the patients to viral infections that, in turn, could trigger

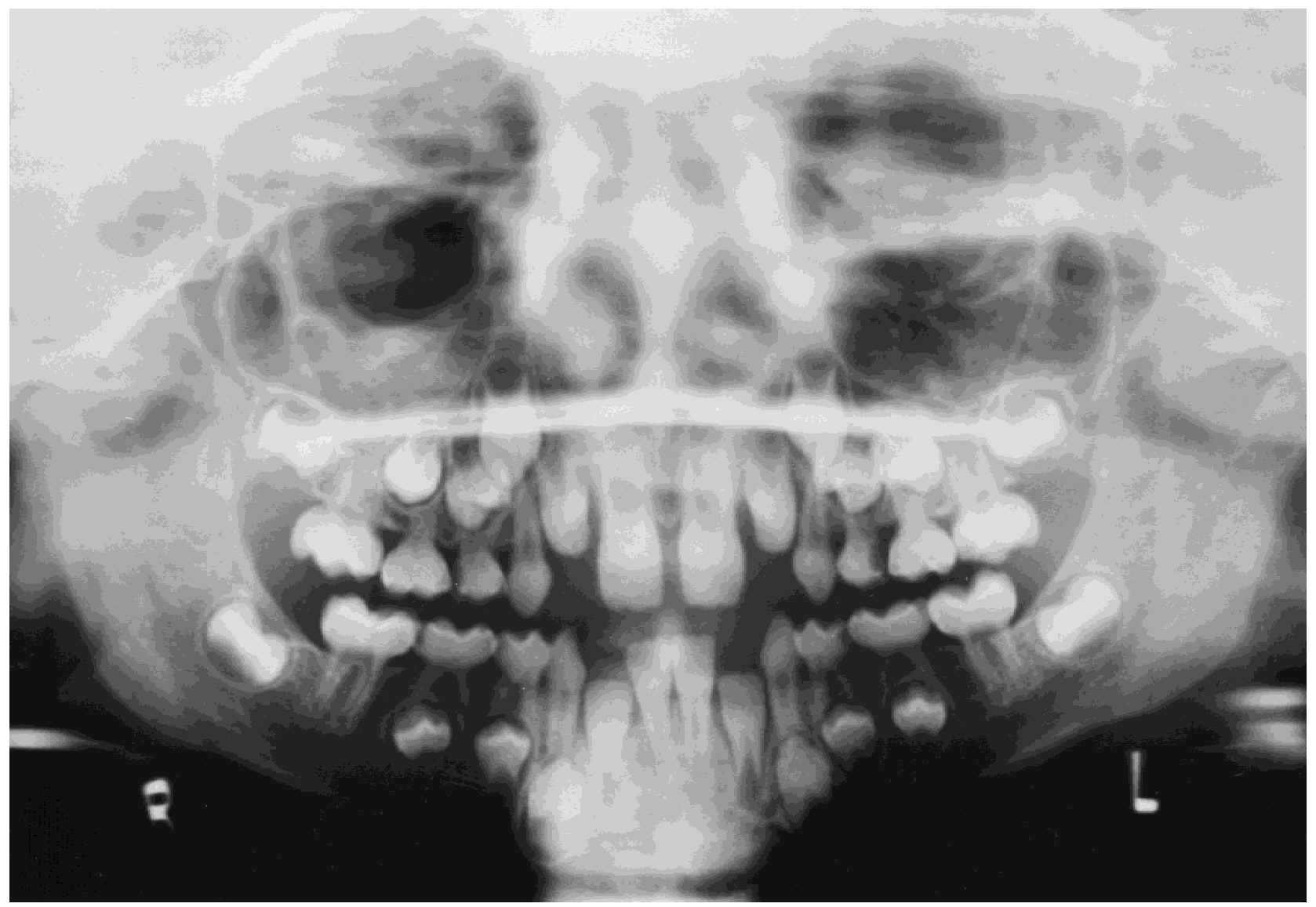

Fig. 1. Panoramic radiograph showing thin roots, bulbous crowns with marked cervical constriction, small pulp chambers, and carious lesions. 
the renal deposition of immune complexes [Spranger et al., 1991], which occurred in this patient. The renal problems are resistant to steroids and immunosuppressive treatment, progressing rapidly, with renal failure 4 to 5 years later [Spranger et al., 1991; Hashimoto et al., 1994; Bayer et al., 1998].

The head and neck characteristics described in the few reported cases include triangular face, broad nasal bridge, bulbous nose tip, small palpebral fissures, short neck, long upper lip, and low hairline [Schmidt et al., 1997; Bayer et al., 1998; Boerkoel et al., 1998; Saraiva et al., 1999]. Furthermore, individuals with del(16q) show orofacial anomalies such as high forehead, broad nasal bridge, hypertelorism, small upturned nose, upslanting palpebral fissures, low-set poorly formed ears, highly arched palate, and micrognathia [Casamassima et al., 1990; Gorlin et al., 1990]. However, no dental abnormalities were reported with del(16q). This is the first report of a patient with SIOD in which dental radiographic findings are discussed. Ludman et al. [1993] described a patient with "microdontia." Those authors postulated that the association of abnormal teeth and a spondyloepiphyseal dysplasia of the Schimke type pointed to a generalized disturbance of the connective tissue matrix. This is in agreement with the tentative diagnosis of DI made for this patient, as it occurs as a primary defect of the collagen matrix that is common to bone and teeth [Stewart et al., 1982; Regezi et al., 1989; Waltimo et al., 1996]. It could also be speculated that the renal problems commonly seen in these patients could possibly lead to dental developmental disturbances, although the patient did not present any staining or incremental defects in the teeth as is usually seen in renal disease. Moreover, the dental anomalies were not caused by immunosuppressive drugs because they were observed before the bone marrow and renal transplants.

DI is an autosomal recessive disorder with a prevalence of about 1:8,000 Caucasians in the United States [Neville et al., 1995]. The teeth have a yellow-brown to blue-gray opalescent discoloration. Fracture of the enamel, tooth wear, short blunt roots, bulbous crowns, and pulpal obliteration are commonly seen, although there can be a significant variation in tooth color and wear [Waltimo et al., 1996]. The disorder has been classified into three types. DI type I is associated with osteogenesis imperfecta, a condition characterized by bone fragility and defects of the collagen molecule [Wright, 1992; Waltimo et al., 1996]. The deciduous teeth are usually more severely affected than the permanent dentition, and the pulp chambers are abnormally large initially, gradually becoming obliterated [Wright, 1992].

In DI type II, also called hereditary opalescent dentin, there is an amber radiolucency of both primary and permanent teeth with considerable variation in expression. The roots are short and thin, and there is accelerated pulp obliteration due to the deposition of abnormal dentin, as observed in this patient (Fig. 1). Although little wear was seen in this case, the enamel usually fractures easily due to poor support provided by the abnormal dentin and the lack of microscopic scalloping that provides a mechanical lock between enamel and dentin [Stewart et al., 1982; Regezi et al., 1989; Neville et al., 1995; Sapp et al., 1997]. An excessive constriction at the cemento-enamel junction gives the teeth a bell or tulip shape. The cementum, supporting the alveolar bone and periodontal ligament, appear normal [Neville et al., 1995; Sapp et al., 1997]. DI type III is considered to be a variant of the two other types, present in the Brandywine isolate of Maryland [Wright, 1996; Sapp et al., 1997]. Thus, it will be important to document dental and oral findings in all cases of Schimke immuno-osseous dysplasia to determine whether the disorder shows dental abnormalities as one of its main characteristics. The affected patients should be referred for a thorough clinical and radiographic oral examination.

\section{REFERENCES}

Bayer M, Baxova A, Olejar T, Kozlowski K. 1998. Schimke immunoosseous dysplasia: a case report. Radiol Med 95:369-371.

Boerkoel CF, Nowaczyk MJM, Blaser SI, Meschino WS, Weksberg R. 1998. Schimke immunoosseous dysplasia complicated by moyamoya phenomenon. Am J Med Genet 78:118-122.

Casamassima AC, Klein RM, Wilmot PL, Brenholz P, Shapiro LR. 1990. Deletion of $16 \mathrm{q}$ with prolonged survival and unusual radiographic manifestations. Am J Med Genet 37:504-509.

Gorlin RJ, Cohen MM, Levin LS. 1990. Syndromes of the Head and Neck. New York: Oxford University Press. 977 p.

Hashimoto K, Takeuchi A, Ieshima A, Takada M, Kasagi M. 1994. Juvenile variant of Schimke immunoosseous dysplasia. Am J Med Genet 49: 266-269.

Jones KL Jr, Bay CA. 1980. Dysmorphic syndromes and their significance to the anesthesiologist. In: Stehling LC, Zauder HL, editors. Implications of congenital anomalies in children. New York: AppletonCentury. pp 9-22.

Lama G, Marrone N, Majorana M, Cirillo F, Salsano ME, Rinaldi MM. 1995. Spondyloepiphyseal dysplasia tarda and nephrotic syndrome in three siblings. Pediatr Nephrol 9:19-23.

Ludman MD, Cole DEC, Crocker JFS, Cohen MM. 1993. Schimke immunoosseous dysplasia: case report and review. Am J Med Genet 47:793796.

Mogeray C, Muralidhar V. 1996. Spondyloepiphyseal dysplasia congenita syndrome: anesthetic implications. Anesth Analg 83:433-434

Neville BW, Damm DD, Allen CM, Bouquot JE. 1995. Oral and maxillofacial pathology. Philadelphia: WB Saunders Co. $711 \mathrm{p}$.

Regezi JA, Sciubba JJ. 1989. Oral pathology: Clinical-pathological correlations. Philadelphia: WB Saunders Co. 615 p.

Santavá A, Zapletalová J, Michálková K, Hanáková S, Kopriva F, Santavy J, Dusek J, Kleinová D. 1994. Spondyloepiphyseal dysplasia with nephrotic syndrome (Schimke immunoosseous dysplasia). Am J Med Genet 49:270-273.

Sapp JP, Eversole LR, Wysocki GP. 1997. Contemporary oral and maxillofacial pathology. St. Louis: CV Mosby. 433 p.

Saraiva JM, Dinis A, Resende C, Faria E, Gomes C, Correia AJ, Gil J, da Fonseca N. 1999. Schimke immuno-osseous dysplasia: case report and review of 25 patients. J Med Genet 36:786-789.

Schmidt B, Christen HJ, Herkenrath P, Benz-Bohm G, Müller-Beghaus J, Querfeld U. 1997. Cerebral complications of Schimke immuno-osseous dysplasia. Eur J Pediatr 156:789-791.

Spranger J, Hinkel GK, Stöss H, Thoenes W, Wargowski D, Zepp F. 1991. Schimke immuno-osseous dysplasia: a newly recognized multisystem disease. J Pediatr 119:64-72.

Stewart RE, Barber TK, Troutman KC, Wei SH. 1982. Pediatric dentistry: scientific foundations and clinical practice. St. Louis: CV Mosby. $1,027 \mathrm{p}$.

Waltimo J, Ojanotko-Harri A, Lukinmaa PL. 1996. Mild forms of dentinogenesis imperfecta in association with osteogenesis imperfecta as characterized by light and transmission electron microscopy. J Oral Pathol Med 25:256-264

Wright JT. 1992. The diagnosis and treatment of dentinogenesis imperfecta and amelogenesis imperfecta. Hell Dent J 2:17-24. 